\title{
Single Center Experience On Colonic Volvulus; Is CT
}

\section{Necessary For Diagnosis?}

\author{
Mesut Aydın ${ }^{1^{\star}}$, Serhat Özer ${ }^{2}$, Yaren Dirik ${ }^{1}$, Sehmus Ölmez ${ }^{3}$, Çetin Kotan ${ }^{4}$, Canan Demir ${ }^{5}$ \\ ${ }^{1}$ Department of Gastroenterology, Van Yuzuncu Yil University, Van, Turkey \\ ${ }^{2}$ Department of Gastroenterology, Artvin State Hospital, Artvin, Turkey \\ ${ }^{3}$ Department of Gastroenterology, Adana Numune Research and Education Hospital, Adana, Turkey \\ ${ }^{4}$ Department of General Surgery, V an Yuzuncu Yil University, Van, Turkey \\ ${ }^{5}$ Van Yuzuncu Yil University, Vocational School of Healthcare, Van, Turkey
}

\begin{abstract}
Colonic volvulus is rare but hazardous cause of bowel obstruction. We tried in this study to assess efficacy of plain $\mathrm{x}$-ray and endoscopic decompression in diagnosis and treatment, respectively.

A total of 34 patients diagnosed with colonic volvulus were retrospectively analyzed and success rates of endoscopic decompression were noted. Descriptive statistics for continuous variables were expressed as Mean \pm Standard Deviation and Median (Min-Max), and it is expressed as number and percentage for categorical variables. Correlations between some parameters in colonic volvulus patients were examined.

A total of 34 patients, $76 \%(n=26)$ male and $24 \%(n=8)$ female, were included in the study. All patients except those with mucosal necrosis underwent successful colonoscopic detorsion $(n=29 ; 85 \%)$. Plain $x$-ray alone was sufficient for diagnosis in $94 \%$.

Colonoscopic detorsion, by gaining time for elective surgery, is an effective treatment modality and computed tomography (CT) is not necessary for diagnosis in colonic volvulus.
\end{abstract}

Key Words: Volvulus, Detortion, Thomography

\section{Introduction}

Colonic volvulus is a cause of acute obstruction secondary to rotation of any segment of colon around its own mesenteric axis (1). It is a rare but hazardous cause of bowel obstruction $(2,3)$. It has been studied for centuries and Hippocrates advocated insufflating huge amount of air and placing a 10 -finger long suppository through anus for treatment of this disorder (4). In the United States, colonic volvulus comprises about 10-15\% of all colonic obstruction cases (5). The most frequent site of volvulus is sigmoid colon in the United States and Western Europe. It is responsible for $20-50 \%$ of all bowel obstructions in Asia, Eastern Europe and under-developed countries (6). In developing countries; high-fiber diet, chronic constipation and prolonged bed rest are considered as predisposing factors (7). Sites of involvement with decreasing frequency are reported as sigmoid colon, cecum, splenic flexure and descending colon. Colonic segment must be long and mobile enough to make volvulus occur. As a result of rotation and obstruction, liquid along with gas accumulate in the obstructed area and may lead to distention, ischemia, gangrene or even perforation (8). Early endoscopic decompression resolves acute obstruction in most cases (9). However, due to high risk of recurrence despite successful early colonoscopic decompression, timely surgical approach is recommended (8).

Volvulus is among emergency situations in gastroenterology. We, although many surgical reports exist, aimed herein to shed light on this issue from a gastroenterological perspective.

\section{Material and Method}

Patients applying to Yuzuncu Yil University Hospital Emergency Room between January 2012 and January 2018 with an established diagnosis of colonic volvulus approached with an initial endoscopy were included in the study.

Diagnoses of patients were established with medical history, physical examination and plain $\mathrm{x}$ rays. Patients were applied endoscopic detorsion

${ }^{*}$ Corresponding Author: Mesut Aydin, Van, Yuzuncu Yil University, Medical Faculty, Department of Gastroenterology, Van, Turkey E-mail: gmstaydin@gmail.com, Telephone: +900(506)6892247 
using Fujinon EC 530 WL video colonoscopy system.

Patients' files and endoscopic records were retrospectively searched. Data on age, gender, previous history of volvulus, white blood cell count, localization of volvulus, time between presentation and colonic detorsion, colonoscopic findings, need for surgical intervention and prognosis were evaluated.

Statistical Analysis: Descriptive statistics for continuous variables were expressed as Mean \pm Standard Deviation and Median (MinMax), and it is expressed as number and percentage for categorical variables. Shapiro-Wilk test was used to test distribution of normality. In paired group comparisons in terms of continuous variables; T-Test was used for normal distribution condition and Mann Whitney $\mathrm{U}$ test was used for cases where normal distribution condition was not provided. In order to determine the relationship between continuous variables, Pearson correlation coefficient was calculated in cases where normal distribution condition was provided between groups and Spearman's rank correlation coefficient was calculated in cases where normal distribution condition was not provided. The statistical significance level was taken as $\mathrm{p}<0.05$ and the SPSS statistical software version 19.0 (SPSS Inc, Chicago, III, USA) pack has used for analyses.

\section{Results}

A total of 34 patients, $76 \%(n=26)$ and $24 \%(n=8)$ of which were male and female, respectively, were included. Median age was 62 (20-92). Median age of males was 67 (20-92) while that of females was 39,50 (23-78) (Table 1). All patients except those with mucosal necrosis $(n=5 ; 15 \%)$, whose procedure was ended immediately, underwent successful colonoscopic detorsion. A second colonoscopic detorsion had to be performed in 5 $(15 \%)$ patients. Time past between presentation and colonoscopy was between 1-43 hours. Volvulus point was sigmoid colon at all patients. $21(62 \%)$ had leukocytosis at the time of diagnosis while 13 did not. According to the results of comparison between age, glucose, ure, kre, ast, alt, wbc, hb and plt for men and women, ast was statistically significant. Accordingly, AST level in women was lower than men (Table 2). As for endoscopic findings, 19 (56\%) patients had normal mucosa while $10(29 \%)$ had hyperemia, edema and erosions and the remaining $5(15 \%)$ did necrotic foci. Table 1 shows colonoscopic findings. Subsequent to colonoscopy, 6 of 19 $(32 \%)$ patients with normal mucosa underwent surgery, while the other 13 did not consent. 9 of $10(90 \%)$ with hyperemia, edema or erosion but necrosis underwent but 1 rejected surgery. 4 of 5 patients $(80 \%)$ with necrosis underwent urgent surgery while the remaining 1 patient died of cardiac arrest during surgical preparation. Overall, all patients were recommended surgery, but $14 \mathrm{did}$ not accept. A total of 2 patients died of cardiac problems, one of which, who was 89 years of age, during surgical preparation and the other with the age of 76 , at sixth post-operative day following sigmoid resection plus Hartmann colostomy.

\section{Discussion}

Colonic volvulus is one of the rare causes of colonic obstructions. It must be handled as a gastroenterological emergency. It is noted in literature that this disorder is more frequent in elderly males. Our study correlates with this finding. As for mucosal assessment in our study, 5 of 34 patients had necrosis. Those with necrosis were found to have a colonoscopy within two hours from presentation. Presence of necrosis despite this relatively quick intervention time is, in our opinion, due to late arrivals of patients at our hospital from different neighbor cities. All patients accepting surgery were electively

operated following endoscopic detorsion. Since the time between starting of symptoms and presentation to our hospital could not be assessed, time needed for necrosis could not be calculated. This is the key limitation of this study. Since the two patients died in this study were elderly and died of cardiologic disorders, we are of the opinion that a cardiology consultation may be of vital importance particularly in elderly patients.

Cecal volvulus was not detected in this study although it is reported between $20-30 \%$ in literature (8). In a recent study including 21 patients and 30 cases from Japan, computed tomography (CT) was used for diagnosis at all patients (10). In our study, $32(94 \%)$ patients had plain $\mathrm{x}$-ray only to establish diagnosis, and coffee bean sign was noted (Figure 1). CT was required in two patients only. Colonoscopies performed in these patients supported the diagnosis, thus; in those with consistent clinical picture, we are of the opinion that plain $\mathrm{x}$-ray is sufficient to establish diagnosis. CT should be reserved for those with 
Table 1. Descriptive Statistics

\begin{tabular}{lc}
\hline Patients & 34 \\
\hline Age & $62(20-92)$ \\
Gender M/F & $26(76 \%) / 8(24 \%)$ \\
Comorbidities & $6(18 \%)$ \\
Mucosa & Normal: $19(56 \%)$ \\
& Hyperemia, edema or erosion: $10(29 \%)$ \\
& Necrosis: $5(15 \%)$ \\
\hline
\end{tabular}

Table 2. Characteristics and comparison results of Male and Female

\begin{tabular}{lccc}
\hline & $\begin{array}{c}\text { Male } \\
\text { Mean } \pm \text { SD } / \text { Median(min-max }) \\
(\mathrm{n}=26)\end{array}$ & $\begin{array}{c}\text { Female } \\
\text { Mean } \pm \text { SD } / \text { Median(min-max }) \\
(\mathrm{n}=8)\end{array}$ & $\mathrm{p}$ \\
\hline AGE & $67(20-92)$ & $39,50(23-78)$ & 0,133 \\
GLUCOSE & $116(85-176)$ & $118,50 \pm 48,264$ & 0,366 \\
URE & $39,38 \pm 13,209$ & $40,38 \pm 15,454$ & 0,860 \\
KRE & $0,78(0,47-2)$ & $0,76(0,57-1,1)$ & 0,503 \\
AST & $27(15-74)$ & $19(15-29)$ & 0,038 \\
ALT & $19(5-57)$ & $13,50(11-22)$ & 0,084 \\
WBC & $10,41 \pm 3,140$ & $13,16 \pm 5,615$ & 0,084 \\
HB & $14,52 \pm 2,362$ & $14,11 \pm 2,309$ & 0,669 \\
PLT & $231576,92 \pm 87386,577$ & $304875,00 \pm 117983,579$ & 0,065 \\
\hline
\end{tabular}

unclear diagnosis.

Despite different treatment modalities, endoscopic detorsion is recommended to be the initial step (11). Early detorsion will - not only - increase mucosal vascularization and prevent necrosis \& perforation, but also gain time for appropriate bowel cleaning before surgery. Colonoscopic detorsion was performed successfully to meet needed time for surgery in all patients except those with mucosal necrosis.

Recurrence rate following endoscopic detorsion is reported between $21 \%$ and $57 \%$ in literature (12). $5(14 \%)$ of our patients had a previous history of colonic volvulus and endoscopic detorsion. These patients were applied endoscopic detorsion once again and in 1, mucosal necrosis was detected. 4 of these were operated and 1 died of cardiac causes during surgical preparation.

In conclusion, early diagnosis and decompression are of vital importance to maintain mucosal integrity and prevent complications in colonic volvulus. However, patients should be recommended surgery following successful endoscopic detorsion due to high recurrence rates. Plain x-ray alone is usually sufficient to establish diagnosis. This finding is particularly crucial in rural areas where CT may not be accessible due to relatively insufficient radiologic facilities.

\section{References}

1. Mulas C, Bruna M, García-Armengol J, Roig JV. Management of colonic volvulus. Experience in 75 patients. Rev Esp Enferm Dig 2010; 102: 239-248.

2. Raveenthiran R, Madiba TE, Atamanalp SS, De U. Volvulus of the sigmoid colon. Colorectal Dis 2010; 12: 1-17.

3. Atamanalp SS, Yildirgan MI, Basoglu M, Kantarci M, Yilmaz I. Sigmoid colon volvulus in children: review of 19 cases. Pediatr Surg Int 2004; 20: 492-495.

4. Ballantyne G H. Review of sigmoid volvulus: history and results of treatment. Dis Colon Rectum 1982; 25: 494-501.

5. Ballantyne G H, Brandner M D, Beart R W, Ilstrup D M. Volvulus of the colon. Incidence and mortality. Ann Surg 1985; 202: 83-92.

6. Hellinger M D Steinhagen R M Colonic volvulus 1st ed. New York: Springer 2009; 286-298.

7. Lau KC, Miller BJ, Schache DJ, Cohen JR. A Study of large-bowel volvulus in urban Australia. Can J Surg 2006; 49: 203-207.

8. Madiba TE, Thomson SR. The management of cecal volvulus. Dis Colon Rectum 2002; 45: 264-267.

9. Grossmann EM, Longo WE, Stratton MD, Virgo KS, Johnson FE. Sigmoid volvulus in 
department of veterans affairs medical centers. Dis Colon Rectum 2000; 43: 414-418.

10. 10-Tomoya I, Suguru N, Shuji S, Haruo S, Hiroyuki K, Hiroshi N. Clinical outcomes of sigmoid colon volvulus: identification of the factors associated with successful endoscopic detorsion. Intest Res 2017; 15: 215-220.

11. Oren D, Atamanalp SS, Aydinli B, et al. An algorithm for the management of sigmoid colon volvulus and the safety of primary resection: experience with 827 cases. Dis Colon Rectum 2007; 50: 489-497.

12. Talavioglu K,Aydın E,Ertekin C,Guloglu R,Kurtoglu M. Our Current Approach In The Treatment of Sigmoid Colon Volvulus. Turkish Journal of Trauma and Emergency Surgery 2002; 8: 102-107. 